\title{
Maturação Física: Uma Revisão da Literatura, com Especial Atenção à Criança Brasileira
}

\section{Physical Maturation: A Review with Special Reference to Brazilian Children}

\author{
Maria de Fátima da S. Duarte ${ }^{1}$
}

DUARTE, M. F. S. Physical Maturation: A Review with Special Reference to Brazilian Children. Cad. Saúde Públ., Rio de Janeiro, 9 (supplement 1): 71-84, 1993.

This article presents an overview of the maturation process in children and discusses data from some Brazilian samples. Data related to secondary sexual characteristics in both sexes are compared to other populations. The national median menarcheal age in Brazilian girls obtained by INAN (1990) was 13.2 years. The menarcheal age of Brazilian athletes is higher than the non-athletic population. The developmental age of breast, genitals, and pubic hair in Brazilian children differ little in relation to European or other Latin American populations. Several studies developed in Brazil show that menarche is a landmark for the process of fat accumulation in females. However, in boys during the sexual maturation process, significant changes in the skinfold thickness amount and distribution are not observed. It is evident that there is a lack of studies focusing on growth, development, and maturation of adolescents in several regions of Brazil, particularly male adolescents.

Key words: Anthropometry; Sex Characteristics; Sex Maturation

\section{INTRODUÇÃO}

Crescimento, desenvolvimento e maturação são processos complexos que levam, no ser humano, cerca de 20 anos antes de se completarem. O primeiro diz respeito a mudanças no tamanho do indivíduo, considerando o corpo como um todo ou partes dele; o segundo, a alterações nas funções orgânicas; e o terceiro, a variações na velocidade e no tempo em que o indivíduo atinge a maturidade biológica. Como mencionado por Tse et al. (1989), o crescimento, nos primeiros anos de vida, é grandemente dependente da nutrição; na idade pré-escolar, particularmente dependente da quantidade de hormônio de crescimento; e na puberdade, resultante da integração entre a ação do hormônio de crescimento e os esteróides sexuais.

${ }^{1}$ University of Illinois at Urbana-Champaign, Department of Kinesiology. 906 South Goodwin Ave. Urbana, Illinois - 61801, U.S.A.
A puberdade é a terceira fase de crescimento somático acelerado, depois da fase intra-uterina e de um pequeno crescimento acelerado na infância (entre 6 e 8 anos de idade), também chamado de crescimento intermediário (Butler et al., 1990; Gasser et al., 1985; 1991; Molinari et al., 1980). Quando na puberdade, o crescimento em estatura chega a 12 e $14 \mathrm{~cm} / \mathrm{ano}^{-1} \mathrm{em}$ meninas e meninos, respectivamente, sendo que as primeiras amadurecem em torno de dois anos mais cedo (Eveleth \& Tanner, 1990).

O presente artigo contém uma revisão da literatura sobre maturação sexual da criança brasileira. Para as meninas, são enfatizados, principalmente, dados relativos às características sexuais secundárias (mamas, pêlos pubianos) e menarca (primeira menstruação); para os meninos, aqueles referentes ao desenvolvimento de genitais, pêlos pubianos e volume testicular. Para ambos os sexos, são discutidas as relações entre antropometria e características sexuais secundárias. 


\section{MATURAÇÃO SEXUAL}

As características sexuais primárias são aquelas relacionadas diretamente com a reprodução. Nas meninas, dizem respeito ao desenvolvimento dos ovários, do útero e da vagina; nos meninos, ao desenvolvimento dos testículos, próstata e produção de esperma. As características sexuais secundárias são as ligadas ao dimorfismo sexual externo, isto é, o desenvolvimento dos seios, pênis, pêlos faciais, pêlos pubianos e modificação da voz.

Os estudos de maturação tendem a se concentrar nas características sexuais secundárias, devido à impossibilidade de determinação do nível de desenvolvimento dos órgãos sexuais internos. Reynolds \& Wine $(1948 ; 1951)$ foram uns dos primeiros autores a estabelecer estágios de maturação de características sexuais secundárias. Como mencionado por Roche et al. (1983), a popularização da avaliação da maturação sexual deveu-se, contudo, a Tanner (1962), que elaborou um sistema de fotografias para a determinação dos estágios de desenvolvimento dos pêlos pubianos para ambos os sexos (estágios de PP1 a PP6), de desenvolvimento mamário para meninas (estágios de M1 a M5) e de desenvolvimento dos genitais para meninos (estágios de G1 a G5). O fato de as fotos apresentadas por Tanner (1962) serem em preto-e-branco representa uma dificuldade na avaliação dos diferentes estágios das características sexuais. As pranchas coloridas publicadas por Van Wieringen et al. (1971) constituem uma alternativa, pois nelas ficam mais visíveis os pêlos pubianos nos estágios iniciais (PP1 e PP2) e os genitais nos estágios G2 e G4, onde a coloração do escroto deve ser considerada.

Além das características sexuais secundárias acima discutidas, pode-se observar também a presença ou não de pêlos axilares em ambos os sexos, a presença de pêlos faciais, mudança de voz, volume dos testículos, bem como a oigarca (primeira ejaculação) nos meninos e a menarca (primeira menstruação) nas meninas.

A seqüência das mudanças maturacionais durante a puberdade é predizível e progressiva, estando o seu início relacionado a fatores genéticos e ambientais (Eveleth \& Tanner, 1990). Em geral, o primeiro sinal do seu início, nos meninos, é o aumento do volume testicular
(Prader, 1966; Zachmann et al., 1974), que principia com $4 \mathrm{ml}$ (Eveleth \& Tanner, 1990; Marshall \& Tanner, 1974; Sempé et al., 1979). As etapas seguintes são: desenvolvimento dos genitais (G); surgimento dos pêlos pubianos (PP); pico de velocidade em estatura; aparecimento dos pêlos axilares; e, finalmente, quando os pêlos pubianos e genitais estão quase no estágio adulto (PP4 e G4 ou G5), surgimento dos pêlos faciais. Em média, os meninos já têm produção de esperma (espermarca) entre 13,4 e 14,0 anos, quando estão com volume testicular em torno de 10-11ml. Nielsen et al. (1986), com base em dados longitudinais, verificaram que a espermarca precedeu o pico de crescimento máximo em estatura em meses. Além disso, foi evidenciada grande variabilidade nos estágios de pilosidade pubiana, tendo sido encontrados meninos com espermarca apresentando desde o estágio 1 até o 5. Infelizmente, os estudos de maturação encontram dificuldades para a determinação da idade da primeira ejaculação, uma vez que ela, muitas vezes, passa despercebida pelo adolescente por ocorrer durante o sono.

Nas meninas, a puberdade principia-se, geralmente, pelo desenvolvimento mamário $(\mathrm{M})$, seguido do aparecimento dos pêlos pubianos, do pico de velocidade em estatura, maior desenvolvimento dos seios e pêlos pubianos, menarca e, finalmente, o estágio adulto de mamas e pêlos pubianos.

O intervalo de tempo entre o início da puberdade e o estágio adulto é bastante variável em ambos os sexos. Nos meninos, o desenvolvimento dos testículos inicia-se aos 11 anos, logo seguido pelo início do desenvolvimento dos genitais. Estima-se em três anos o período médio de desenvolvimento desde o estágio 2 ao 5 de genitais e pêlos pubianos (Lee, 1980; Marshall \& Tanner, 1970; Taranger et al., 1976). No caso das meninas, é de 3 a 4 anos o período médio entre os estágios iniciais de desenvolvimento das mamas (M2) e pêlos pubianos (PP2) e o estágio adulto (Billewicz et al., 1981b; Largo \& Prader, 1983a, 1983b; Marshall \& Tanner, 1969).

O processo de desenvolvimento das características sexuais secundárias ocorre de maneira mais rápida nos estágios iniciais. Por exemplo, as mudanças do estágio de mamas 2 para 3, ou 
do estágio de pêlos pubianos 2 para 3, levam em torno de um ano, tornando-se o processo mais lento à medida que o adolescente aproxima-se do estágio adulto. Esses valores são médias observadas em populações estrangeiras, o que impede sua utilização universal, devido a diferenças populacionais e ambientais.

Recentemente, tem sido discutido o problema da invasão de privacidade na avaliação das características sexuais secundárias, que nem sempre é aceita e viável, dependendo das condições locais, humanas e, evidentemente, do consentimento do sujeito ao exame clínico. Uma alternativa é a auto-avaliação, procedimento já validado em adolescentes norte-americanos (Duke et al., 1980; Kozinetz, 1988; Morris \& Udry, 1980), franceses (Lopez et al., 1988) e brasileiros (Matsudo \& Matsudo, 1991; Saito, 1984). Estes estudos mostram correlações altas $(r=0,80)$ entre a auto-avaliação e a aquela feita por profissional especializado. É possível que as correlações não sejam mais elevadas devido à ocorrência de uma super ou subestimativa dos estágios pelos adolescentes, por motivos culturais ou de auto-imagem

\section{MENARCA}

A maturação sexual de meninas já foi estudada em diversas populações brasileiras, sendo a idade de menarca um dos parâmetros mais freqüentemente reportados (Tabela 1). Vale ressaltar que a maior parte destes estudos baseia-se em pequenas amostras e no método retrospectivo, ou seja, a menina deveria lembrar quando (mês e ano) ocorreu a primeira menstruação.

Os dados nacionais (Tabela 1) evidenciam considerável variabilidade quanto à idade de menarca, variando de 12,2 anos, para amostras de meninas de Guarulhos e São Caetano do Sul (Matsudo, 1982a), a 13,98 anos, em meninas oriundas de Monte Belo (Antunes et al. 1984). Esta variabilidade está possivelmente relacionada a fatores ambientais (clima, relevo geográfico), genéticos, sociais (nutrição, nível sócio-econômico, número de filhos na família) e treinamento físico, entre outros. Riehmer \& Violato (1983) e Silva et al. (1982), por exemplo, ao analisarem a relação entre menarca e tamanho da família em escolares de São Luís (MA) e Rolândia (PR), respectivamente, evidenciaram uma relação direta entre estas variáveis, isto é, quanto maior o tamanho da família, mais tardia a menarca. Os autores especularam que o nível sócio-econômico poderia explicar este fenômeno, ou seja, haveria um déficit nutricional e, com isso, um atraso no processo de maturação. Colli (1985), por sua vez, observou que meninas de alto nível sócio-econômico de Santo André apresentaram menarca mais cedo quando comparadas com aquelas de menor nível da mesma localidade. Em geral, os resultados obtidos no Brasil estão de acordo com os evidenciados em estudos conduzidos em outros países, que indicam que déficits nutricionais podem causar atraso na maturação sexual e no crescimento físico (Galler et al., 1985; Satyanarayana \& Nadamuni Naidu, 1979; Satyanarayana et al., 1980).

Em 1989, o Instituto Nacional de Alimentação e Nutrição do Ministério da Saúde (Inan, 1990) realizou a Pesquisa Nacional sobre Saúde e Nutrição (PNSN), que baseou-se em uma amostragem estrato-randomizada de $62 \mathrm{mil}$ pessoas de todas as regiões do país. Entre outras avaliações, a presença ou não de menarca foi verificada pelo método de status quo, ou seja, a menina respondia se já a havia tido ou não. A idade mediana de menarca obtida neste estudo foi de 13 anos e 2 meses. Infelizmente, a documentação da PNSN não provém informações detalhadas a respeito do grupo etário considerado para fins de cálculo. Com base nos formulários originais da pesquisa, pode-se observar que a pergunta sobre a presença ou não de menarca restringiu-se às mulheres entre 10 e 19 anos. Para aquelas com mais de 20 anos, perguntou-se apenas a idade em que a menarca havia ocorrido. Com isso, as meninas que apresentaram a menarca antes de 10 anos de idade ficaram excluídas da amostra, sugerindo que o valor de 13,2 anos pode estar ligeiramente superestimado.

A idade de menarca pode também ser influenciada pelo nível de treinamento físico. Em geral, atletas têm idade de menarca mais tardia que as meninas não-atletas. Este fenômeno está provavelmente relacionado não somente ao treinamento físico regular, mas também à seleção para atividades esportivas de meninas. 
TABELA 1. Idade de Menarca em Amostras de Meninas Brasileiras

\begin{tabular}{|c|c|c|c|c|}
\hline Autor(es) & Região & $\mathrm{n}$ & Média & $\mathrm{DP}$ \\
\hline INAN (1990) & $\begin{array}{l}\text { Brasil } \\
\text { Região Norte }\end{array}$ & $* * *$ & $* 13,20$ & \\
\hline Silva et al. (1982) & $\begin{array}{l}\text { São Luís/MA } \\
\text { Região Centro-Oeste }\end{array}$ & 302 & 12,40 & 1,20 \\
\hline Bezerra et al. (1973) & $\begin{array}{l}\text { Sobradinho/DF } \\
\text { Região Sudeste }\end{array}$ & 316 & 12,50 & nd \\
\hline Hegg \& Luongo (1976) & São Paulo/SP & 333 & 12,56 & nd \\
\hline Hegg \& Levy (1977) & São Paulo/SP & 1850 & 12,27 & 1,15 \\
\hline Colli (1985) & Santo André/SP & 2584 & ${ }^{* *} 12,60$ & \\
\hline \multirow{3}{*}{ Matsudo (1982a) } & Guarulhos/SP & 54 & 12,20 & nd \\
\hline & São Caetano do Sul/SP & 55 & 12,20 & nd \\
\hline & São Bernardo do Campo/SP & 28 & 12,40 & nd \\
\hline Duarte \& Duarte (1989) & São Caetano do Sul/SP & 210 & 12,35 & 1,25 \\
\hline Campos et al. (1990) & Ilha Bela/SP & 271 & 12,77 & 1,33 \\
\hline Duarte \& Duarte (1992) & São Paulo/SP & 74 & 12,21 & 1,26 \\
\hline \multirow{15}{*}{ Antunes et al. (1984) } & Alfenas/MG & 90 & 12,88 & 1,10 \\
\hline & Perdões/MG & 42 & 12,93 & 1,32 \\
\hline & Areado/MG & 34 & 12,69 & 1,21 \\
\hline & Poços de Caldas/MG & 59 & 12,95 & 1,31 \\
\hline & Cabo Verde/MG & 40 & 13,30 & 1,14 \\
\hline & Santa Rita de Caldas/MG & 22 & 12,86 & 0,91 \\
\hline & Muzambinho/MG & 70 & 12,84 & 1,38 \\
\hline & Monte Belo/MG & 20 & 13,98 & 1,25 \\
\hline & Botelhos/MG & 18 & 13,05 & 1,31 \\
\hline & Nova Rezende/MG & 32 & 13,22 & 0,98 \\
\hline & Carmo do Rio Claro/MG & 47 & 13,35 & 1,23 \\
\hline & Guaxupé/MG & 45 & 13,41 & 1,11 \\
\hline & Belo Horizonte/MG & 32 & 13,57 & 1,74 \\
\hline & Lavras/MG & 81 & 12,87 & 1,15 \\
\hline & Região Sul & & & \\
\hline Violato \& Matsudo (1983) & Rolândia/PR & 440 & 13,20 & nd \\
\hline Riehmer \& Violato (1983) & Londrina/PR & & 12,50 & nd \\
\hline Petroski et al. (1983) & Florianópolis/SC & 146 & 12,93 & 1,20 \\
\hline \multirow[t]{3}{*}{ De Bem \& Petroski (1988) } & Santa Catarina & & & \\
\hline & Região Serrana & 209 & 12,83 & 1,06 \\
\hline & Região Litorânea & 294 & 12,45 & 1,04 \\
\hline
\end{tabular}

* Idade de menarca calculada por mediana - método status quo

** Idade de menarca calculada por probito

nd = não disponível

*** Provavelmente em torno de 7.087 sujeitos

com predominância de linearidade corporal, as quais, por sua vez, apresentam menor percentual de gordura.

A Tabela 2 apresenta as médias de idade de menarca em amostras de atletas brasileiras. Os valores são, em geral, mais elevados que a média nacional de 13,2 anos evidenciada pela PNSN (Inan, 1990). As meninas que praticam atletismo e ginástica olímpica são aquelas que apresentam os valores mais elevados de idade de menarca. São exceções as nadadoras e as jogadoras de voleibol, que, em geral, apresentam idade de menarca quase idêntica à da população brasileira geral.

\section{Comparações com outras Populações}

É difícil comparar valores de idade de menarca entre populações devido a uma série de fatores, tais como: metodologia utilizada, ano de realização da pesquisa, condições climáticas, altitude, nível sócio-econômico da região 
TABELA 2. Idade de Menarca em Amostras de Atletas Brasileiras

\begin{tabular}{llccc}
\hline \hline Autor(es) & Modalidade & $\mathrm{n}$ & Média & DP \\
\hline Mendes \& Matsudo (1980) & Atletismo - COTP & 12 & 13,68 & nd \\
& Atletismo - Camp. Sul-Americano & 14 & 13,97 & nd \\
Matsudo \& Sessa (1980) & Atletismo - COTP & nd & 14,10 & nd \\
Hegg et al. (1982) & Atletismo - Camp. Sul-Americano & 18 & 13,90 & 1,77 \\
Matsudo (1982b) & Atletismo & 12 & 14,50 & 3,13 \\
Matsudo \& Sessa (1980) & Basquetebol - COTP & nd & 13,00 & nd \\
Matsudo (1982b) & Basquetebol & 9 & 13,03 & 2,17 \\
Benito et al. (1983) & Basquetebol - 44 Jog. Abertos de Araçatuba & 53 & 13,81 & 1,56 \\
& Basquetebol - Seleção Brasileira & 17 & 13,74 & 2,02 \\
Matsudo \& Sessa (1980) & Ginástica Olímpica - COTP & nd & 14,60 & nd \\
Matsudo (1982b) & Ginástica Olímpica & 4 & 14,60 & 0,63 \\
Vívolo et al. (1983) & Ginástica Rítmica Desportiva - Sel. Paulista & 10 & 12,75 & 0,54 \\
Matsudo \& Sessa (1980) & Natação - COTP & nd & 13,50 & nd \\
Matsudo (1982b) & Natação & nd & 13,51 & 1,71 \\
Silva et al. (1984) & Natação & 53 & 12,57 & 1,16 \\
Matsudo \& Sessa (1980) & Voleibol - COTP & nd & 12,80 & nd \\
Matsudo (1982b) & Voleibol & 14 & 12,84 & 0,63 \\
\hline \hline
\end{tabular}

DP $=$ Desvio Padrão nd = Não disponível

COTP = Centro Olímpico de Treinamento e Pesquisa - SP

estudada; além da própria natureza da investigação (se transversal ou longitudinal).

Ao comparar-se as médias de idade de menarca em meninas de diversos países, pode-se observar que os valores para as brasileiras são inferiores àqueles de meninas de diversos países desenvolvidos, mas semelhantes àqueles obtidos nos E.U.A. e Japão (Tabela 3). Em relação aos países latino-americanos, as brasileiras apresen- tam idade de menarca semelhante às das argentinas, bolivianas, cubanas e venezuelanas.

A tendência de redução da idade de menarca é um fenômeno universal que vem sendo observado há quase 150 anos tanto em países desenvolvidos como naqueles em desenvolvimento. Chamado de tendência secular, este fenômeno parece dever-se a melhorias nas condições sanitárias, alimentares e habitacio-

TA BELA 3. Idade de Menarca em Amostras de Meninas de Outros Países em Comparação aos Dados da População Brasileira (Inan, 1990)

\begin{tabular}{|c|c|c|c|c|}
\hline Autor(es) & País & $\mathrm{n}$ & Média & $\mathrm{DP}$ \\
\hline INAN (1990) & Brasil & $* * *$ & ${ }^{*} 13,20$ & \\
\hline Cameron et al. (1991) & África do Sul & 230 & ${ }^{*} 14,03$ & 1,25 \\
\hline Lejarraga et al. (1980) & Argentina & 504 & 12,50 & 0,05 \\
\hline \multirow[t]{2}{*}{ Greksa (1990) } & Bolívia (Desc. Européia) & 455 & ${ }^{*} 13,10$ & nd \\
\hline & (Desc. Aymara) & 375 & * 13,40 & nd \\
\hline \multirow[t]{3}{*}{ Lin et al. (1992) } & China & & & \\
\hline & Han (Urbana) & 84.652 & ${ }^{*} 13,17$ & 1,38 \\
\hline & Han (Rural) & 78.250 & ${ }^{*} 13,83$ & 1,27 \\
\hline Jordan (1985) & Cuba & 13.143 & 13,00 & nd \\
\hline MacMahon (1973) & E.U.A. & 10.291 & 12,76 & 1,41 \\
\hline Billewicz et al. (1981a) & Inglaterra & 699 & 13,37 & 1,14 \\
\hline Marshall \& Tanner (1969) & Inglaterra & 192 & 13,50 & nd \\
\hline Hoshi \& Kouchi (1981) & Japão & 284 & 12,40 & 0,95 \\
\hline Pawson (1977) & Nepal & 286 & 18,10 & nd \\
\hline Largo \& Prader (1983a) ${ }^{* *}$ & Suécia & 142 & 13,40 & 1,10 \\
\hline Taranger et al. (1976) & Suíça & 90 & 13,03 & nd \\
\hline Contreras et al. (1981) & Venezuela & nd & 12,70 & nd \\
\hline
\end{tabular}

DP = Desvio Padrão

${ }^{*}$ Método status quo nd = Não disponível

${ }^{* *}$ Estudo longitudinal
${ }^{* * *}$ Provavelmente em torno de 7.087 sujeitos 
nais, bem como ao controle mais efetivo de doenças.

Aparentemente, a tendência secular de redução da idade de menarca está ocorrendo também no Brasil. Os artigos publicados entre 1912 e 1937, revisados por Hegg \& Levy (1977), reportam valores em torno de 14 anos; portanto, superiores à idade de 13,2 anos evidenciada pela PNSN para a população brasileira na década de 80 (Inan, 1990).

\section{ESTÁGIO MAMÁRIO, GENITAL E DE PÉLOS PUBIANOS}

A Tabela 4 apresenta os dados de estágio mamário e pilosidade pubiana de meninas de diversos países. Nela o Brasil é representado pelo trabalho de Colli (1985), que reporta dados de adolescentes da cidade de Santo André, na Grande São Paulo.
As adolescentes de Santo André iniciam o desenvolvimento das mamas e pêlos pubianos praticamente na mesma idade que as adolescentes de outros países (Tabela 4). Quanto à progressão do desenvolvimento mamário, as brasileiras parecem amadurecer mais tarde, o que não pode ser totalmente confirmado, já que o estudo de Colli (1985) foi transversal. O desenvolvimento de pêlos nas meninas brasileiras parece ocorrer dentro das mesmas faixas etárias dos outros estudos.

Os meninos brasileiros parecem iniciar o desenvolvimento dos genitais em torno da mesma idade dos adolescentes de outros países. Atingem, contudo, os estágios adultos um pouco mais tardiamente. Quanto ao início do desenvolvimento da pilosidade pubiana, parece haver uma certa semelhança nas idades de início e chegada ao estágio adulto entre os diversos estudos (Tabela 5).

TABELA 4. Estágios de Mama e Pêlos Pubianos em Amostras de Meninas do Brasil e de Outros Países

\begin{tabular}{|c|c|c|c|c|c|c|c|c|c|c|}
\hline Autor(es) & País & & M2 & M3 & M4 & M5 & PP2 & PP3 & PP4 & PP5 \\
\hline \multirow[t]{2}{*}{ Colli (1985) } & \multirow[t]{2}{*}{ Brasil } & Média & 11,3 & 12,1 & 14,3 & 16,0 & 11,5 & 12,1 & 13,3 & 15,8 \\
\hline & & DP & 1,0 & 1,1 & 2,2 & 2,1 & 1,2 & 1,1 & 1,7 & 2,1 \\
\hline Avendano \& Valenzuela (1988) & Chile & Média & 10,3 & 11,4 & 12,5 & 13,6 & 10,6 & 11,8 & 12,5 & 13,6 \\
\hline Jordan (1985) & Cuba & Média & 10,8 & 12,3 & 14,0 & nd & 11,5 & 12,7 & 14,2 & nd \\
\hline \multirow[t]{2}{*}{ Lee $(1980)^{*}$} & \multirow[t]{2}{*}{ E.U.A. } & Média & 11,2 & 12,4 & 13,1 & 14,5 & 11,9 & 12,7 & 13,4 & 14,6 \\
\hline & & DP & 1,6 & 1,2 & 0,7 & 1,6 & 1,5 & 0,5 & 1,2 & 1,1 \\
\hline Villarreal et al. (1989) & E.U.A. & Média & 11,0 & 12,2 & 13,9 & 15,1 & 11,3 & 12,4 & 14,1 & 15,5 \\
\hline Dacou-Voutetakis et al. (1983) & Grécia & Média & 10,6 & 11,8 & 12,2 & 14,2 & 10,5 & 11,6 & 12,6 & 14,3 \\
\hline \multirow[t]{2}{*}{ Billewicz et al. (1981b) ${ }^{*}$} & \multirow[t]{2}{*}{ Inglaterra } & Média & 10,8 & 12,0 & 13,1 & 14,0 & nd & nd & nd & nd \\
\hline & & DP & 1,6 & 1,3 & 1,3 & 1,6 & nd & nd & nd & nd \\
\hline \multirow[t]{2}{*}{ Marshall \& Tanner (1969) ${ }^{*}$} & \multirow[t]{2}{*}{ Inglaterra } & Média & 11,2 & 12,2 & 13,1 & 15,3 & 11,7 & 12,4 & 13,0 & 14,4 \\
\hline & & DP & 1,1 & 1,1 & 1,2 & 1,7 & 1,2 & 1,1 & 1,1 & 1,1 \\
\hline \multirow[t]{2}{*}{ Belmaker (1982) } & \multirow[t]{2}{*}{ Israel } & Média & 10,3 & 11,0 & nd & nd & 10,6 & 11,4 & nd & nd \\
\hline & & DP & 1,0 & 1,3 & nd & nd & 1,3 & 1,0 & nd & nd \\
\hline \multirow[t]{2}{*}{ Largo \& Prader (1983b) ${ }^{*}$} & \multirow[t]{2}{*}{ Suécia } & Média & 10,9 & 12,2 & 13,2 & 14,0 & 10,4 & 12,2 & 13,0 & 14,0 \\
\hline & & DP & 1,2 & 1,2 & 0,9 & 1,2 & 1,2 & 1,2 & 1,1 & 1,3 \\
\hline Taranger et al. (1976) & Suíça & Média & 11,0 & 11,8 & 13,1 & 15,6 & 11,5 & 12,0 & 12,9 & 15,2 \\
\hline
\end{tabular}

M = Estágio de mama segundo Tanner (1962)

DP = Desvio Padrão

* Estudo Longitudinal
PP = Estágio de pêlo pubiano segundo Tanner (1962)

nd = Não disponível 
TABELA 5. Estágios de Genitais e Pêlos Pubianos em Amostras de Meninos do Brasil e de outros Países

\begin{tabular}{|c|c|c|c|c|c|c|c|c|c|c|}
\hline Autor(es) & País & & G2 & G3 & G4 & G5 & PP2 & PP3 & PP4 & PP5 \\
\hline \multirow[t]{2}{*}{ Colli (1985) } & \multirow[t]{2}{*}{ Brasil } & Média & 12,0 & 13,2 & 14,7 & 16,9 & 12,5 & 13,5 & 15,3 & 16,2 \\
\hline & & DP & 1,3 & 1,3 & 1,5 & 1,6 & 1,2 & 1,2 & 1,8 & 1,6 \\
\hline Avendano \& Valenzuela (1988) & Chile & Média & 11,1 & 11,9 & 13,1 & 14,2 & nd & nd & nd & nd \\
\hline \multirow[t]{2}{*}{ Muzzo et al. (1988) } & \multirow[t]{2}{*}{ Chile } & Média & 11,8 & 12,9 & 13,9 & nd & 12,0 & 13,1 & 14,0 & nd \\
\hline & & DP & 1,3 & 1,0 & 1,1 & nd & 1,1 & 1,2 & 1,1 & nd \\
\hline Jordan (1985) & Cuba & Média & 11,8 & 13,6 & 14,8 & nd & 12,7 & 14,1 & 15,0 & nd \\
\hline \multirow[t]{2}{*}{ Lee $(1980)^{*}$} & \multirow[t]{2}{*}{ E.U.A. } & Média & 11,9 & 13,2 & 14,3 & 15,1 & 12,3 & 13,9 & 14,7 & 15,3 \\
\hline & & DP & 1,1 & 0,8 & 0,8 & 1,1 & 0,8 & 0,9 & 0,9 & 0,8 \\
\hline Villarreal et al. (1989) & E.U.A. & Média & 12,4 & 13,5 & 14,6 & 16,3 & 12,8 & 13,6 & 14,6 & 16,1 \\
\hline \multirow[t]{2}{*}{ Marshall \& Tanner (1970) } & \multirow[t]{2}{*}{ Inglaterra } & Média & 11,6 & 12,9 & 13,8 & 14,9 & 13,4 & 13,9 & 14,4 & 15,2 \\
\hline & & DP & 1,1 & 1,0 & 1,0 & 1,1 & 1,1 & 1,1 & 1,1 & 1,1 \\
\hline Taranger et al. (1976) ${ }^{*}$ & Suíça & Média & 12,2 & 13,1 & 14,0 & 15,1 & 12,5 & 13,4 & 14,1 & 15,5 \\
\hline
\end{tabular}

$\mathrm{G}=$ Estágio de genitais segundo Tanner (1962)

DP = Desvio Padrão

* Estudo Longitudinal

Tanto para as meninas como para os meninos, poucos estudos fornecem valores médios de idade para o estágio 6 de pilosidade pubiana (distribuição dos pêlos na linha alba).

\section{MATURAÇÃO SEXUAL E ANTROPOMETRIA}

\section{Sexo Feminino}

Observa-se que, ao redor de 6 meses a um ano antes da menarca, já ocorre o pico de velocidade de crescimento em estatura e massa corporal. Há casos, contudo, em que o pico de velocidade de ganho de peso coincide com a menarca. $\mathrm{O}$ peso tende ainda a aumentar após a menarca, devido ao crescimento e desenvolvimento geral do corpo e, principalmente, devido ao aumento nos depósitos de gordura, em função da maior atuação do estrógeno e da progesterona. Por ocasião da primeira menstruação, cerca de $95 \%$ da estatura adulta já foram atingidos e o crescimento linear entra em processo de desaceleração, cessando em torno de 2,5 a 3 anos depois.

As hipóteses levantadas por Frisch \& Revelle
PP = Estágio de pêlo pubiano segundo Tanner (1962) nd $=$ Não disponível

(1969; 1970; 1971) e Frisch et al. (1973), de que uma determinada massa corporal $(39 \mathrm{~kg})$ e uma certa proporção entre gordura e massa corporal magra seriam os desencadeadores metabólicos da menarca, têm sido constantemente refutadas na literatura (Johnston et al., 1975).

Estudos conduzidos no Brasil tampouco têm provido suporte para as hipóteses de Frisch e colaboradores. Saito (1985), por exemplo, analisou dados de idade de menarca e diversas variáveis antropométricas (massa corporal, estatura e dobra cutânea tricipital) em adolescentes de Santo André (SP), visando testar a hipótese de um valor de massa corporal crítico. Enquanto o grupo com menarca mostrou valores superiores para diversas variáveis antropométricas, e das pregas cutâneas em particular, os dados não proveram suporte para a idéia de um valor crítico de massa corporal ou de estatura na época da menarca.

Matsudo (1979) avaliou as dobras cutâneas em escolares de São Caetano do Sul (SP), subdividas em dois grupos para efeito do estudo: com e sem menarca. Foram encontrados valores estatisticamente superiores no somatório das 7 dobras cutâneas (bicipital, tricipital, 
subescapular, supra-ilíaca, axilar média, abdominal e panturrilha) para o primeiro grupo. Analisando dados de dobras cutâneas de um grupo de 543 adolescentes, Hegg \& Hegg (1980) relataram que meninas com menarca (púberes) possuíam valores de dobras cutâneas significativamente maiores que as não-púberes.

Visando avaliar o quanto a menarca poderia influenciar a massa corporal, a estatura, o índice de massa corporal (massa corporal dividida pela estatura ao quadrado) e as dobras cutâneas (tricipital, subescapular e supra-ilíaca), M. Duarte (1987) analisou os dados de escolares de Diadema (SP) com idade cronológica de 12 anos. $\mathrm{O}$ autor constatou que as meninas com menarca possuíam valores superiores para os parâmetros antropométricos em relação àquelas que não haviam experienciado a menarca. A menarca parece ter sido responsável, em um curto espaço de tempo, por importantes transformações nas dimensões e na composição corporal. Isto pôde ser deduzido porque as meninas com menarca foram medidas cerca de apenas 2 meses após a menarca propriamente dita, que ocorreu, em média, aos 12,17 anos.

Em um estudo mais extenso, M. Duarte
(1986) estudou a relação entre as características sexuais secundárias (estágios de mamas e pêlos pubianos, segundo critérios de Tanner (1962) e dobras cutâneas (tricipital, subescapular, suprailíaca e abdominal) em 3.338 escolares de 10 a 19 anos de idade da cidade de Santo André (SP). Aquelas no estágio 5 de mamas (M5) apresentaram valores do somatório de dobras estatisticamente superiores àqueles das meninas menos avançadas (M1, M2, M3 e M4) (Figura 1). Não se verificou, contudo, diferenças no somatório das dobras cutâneas entre as meninas de diferentes idades, mas no mesmo estágio de desenvolvimento das mamas.

Em relação ao desenvolvimento de pelos pubianos, entre as idades de 11 a 15 anos, as médias das dobras cutâneas mostraram-se consistente e estatisticamente superiores para as meninas mais maduras (PP5) (Figura 2). Ao se comparar as meninas de diferentes idades, mas no mesmo estágio de pilosidade pubiana, não se observaram diferenças significativas na média das quatro dobras cutâneas. Em suma, na amostra de meninas de Santo André, foi o estágio de maturação, e não a idade cronológica, que determinou a quantidade de gordura subcutânea.

FIGURA 1. Média de Quatro Dobras Cutâneas em Função do Estágio de Mamas numa Amostra de Meninas Brasileiras. Adaptado de Duarte (1986)

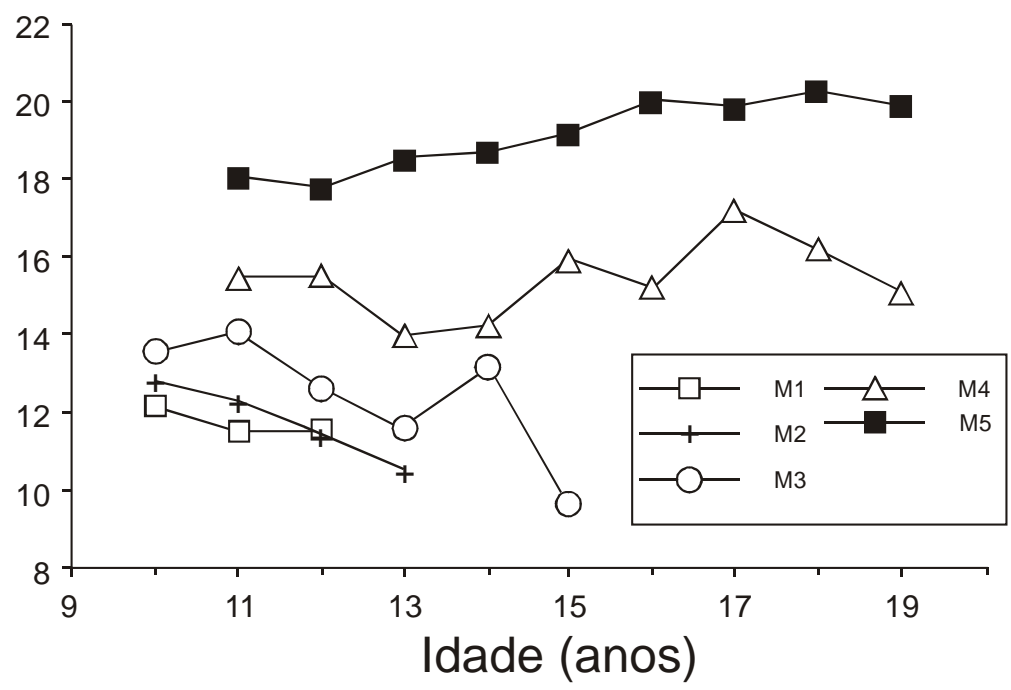


FIGURA 2. Média de Quatro Dobras Cutâneas em Função do Estágio de Pêlos Pubianos numa Amostra de Meninas Brasileiras. Adaptado de Duarte (1986)

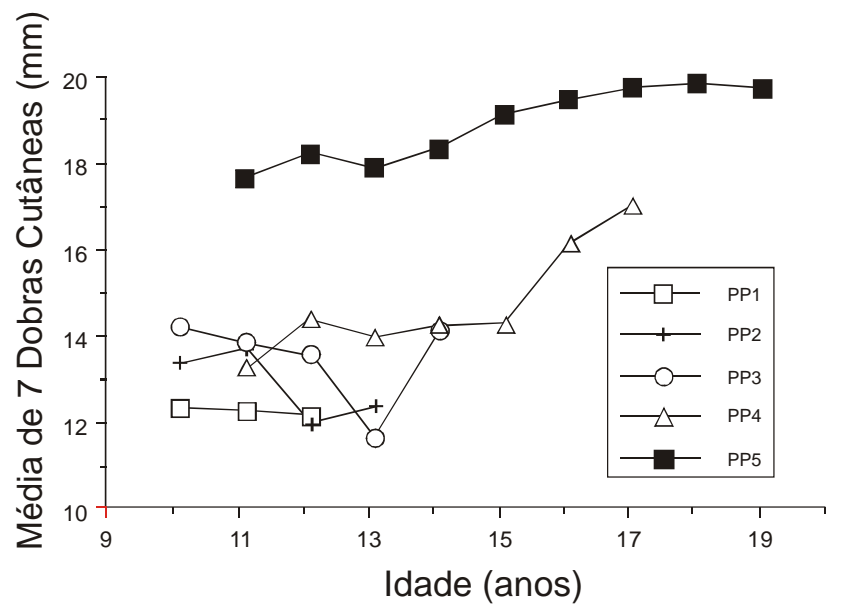

\section{Sexo Masculino}

Os estudos relacionando maturação física e características antropométricas de adolescentes masculinos brasileiros são relativamente escassos e apresentam resultados até certo ponto contraditórios.

Guedes (1981a) comparou o somatotipo de três grupos de meninos com idade cronológica de 14 anos apresentando diferente distribuição de pêlos axilares (ausência, presença parcial ou total). O fato de não terem sido observadas diferenças entre os diversos grupos levou o autor a concluir que a escolha da pilosidade axilar não foi um bom critério para se diagnosticar diferenças em somatotipo. Em um estudo posterior, contudo, o mesmo autor (Guedes, 1981b) encontrou diferenças estatisticamente significantes nas variáveis massa corporal e estatura entre meninos sem e com presença total de pêlos axilares.

Os dados analisados por Bonjardim (1984), referentes a escolares (divididos entre pré-púberes, púberes e pós-púberes) de Santo André, indicaram diferenças estatisticamente significantes para as duas variáveis antropométricas estudadas (comprimento tronco-cefálico e comprimento dos membros inferiores), de acordo com a progressão da maturação sexual (volume testicular, desenvolvimento de genitais e pilosidade pubiana).

A análise de valores das dobras cutâneas (tricipital, subescapular e supracrista-ilíaca) de grupos de escolares de Diadema sem e com pilosidade axilar não indicou diferenças intergrupais (C. Duarte, 1987). Tampouco foram observadas diferenças estatisticamente significantes nas médias de três dobras cutâneas (tricipital, subescapular e supracrista-ilíaca) de escolares de 11 a 14 anos de idade da cidade de São Paulo que diferiam quanto aos estágios de pilosidade pubiana (PP2 a PP5) (Duarte \& Duarte, 1988).

Duarte (1986) analisou os dados de 3.381 escolares de Santo André (SP) com relação aos estágios de genitais, pêlos pubianos, volume testicular e dobras cutâneas (tricipital, subescapular, supracrista-ilíaca e abdominal). Não se observaram diferenças nas médias das dobras entre os meninos em diferentes estágios de maturação. Considerada isoladamente, a dobra tricipital mostrou-se menor naqueles indivíduos menos desenvolvidos em termos de pilosidade pubiana, genitais ou volume testicular, o que não foi o caso das dobras do tronco. Os resultados do estudo indicaram também que não foram os estágios de maturação que determinaram a quantidade de gordura, mas a idade cronológica.

\section{CONCLUSÃO}

Esta revisão indica que são pouco conhecidas as inter-relações entre crescimento, desenvolvimento físico e maturação das crianças brasilei- 
ras. Carece-se de informações também quanto às relações entre idade biológica e os picos de velocidade de crescimento físico.

Sobre o grupo masculino, praticamente não há dados de abrangência nacional, uma vez que os estudos restringem-se a região Sudeste do país. Possivelmente, isto está associado ao fato de ser mais difícil realizar estudos sobre maturação em meninos, já que, para este grupo, não existe um fenômeno correspondente à menarca, parâmetro freqüentemente utilizado para avaliar a maturação no sexo feminino. Estudos sobre o desenvolvimento das características sexuais primárias e secundárias precisam ser realizados para que se tenha dados referenciais mais abrangentes.

A idade de menarca nas meninas brasileiras parece variar entre as diversas regiões do país. Parte desta variação pode ser creditada a diferenças de nível sócio-econômico e estado nutricional. No Brasil, é necessário que sejam realizados estudos abrangentes que levem em consideração todos estes fatores, a fim de que se possa explicar a grande variabilidade intra-regional encontrada na idade de menarca. Seria fundamental a realização de investigações sobre tendência secular, cujos resultados poderiam prover subsídios para se avaliar a melhora ou não das condições de saúde da população.

A menarca parece ser um marco importante no aumento dos depósitos de gordura nas meninas brasileiras. Nos meninos, parece não haver modificações muito marcadas nas dobras cutâneas durante o processo de maturação sexual. A nível de avaliação da quantidade e distribuição de gordura em meninas durante o processo pubertário, deve-se considerar o estágio das características sexuais secundárias nas respectivas análises e comparações.

Deste modo, fica evidenciada a necessidade de se realizarem estudos transversais e longitudinais no país com o intuito de melhor visualizar o fenômeno maturacional no adolescente brasileiro.

\section{AGRADECIMENTOS}

A autora gostaria de agradecer os comentários de Carlos Roberto Duarte e Miguel Angelo Marini ao texto preliminar, aos revisores pelas valiosas sugestões e a Luiz Antonio dos Anjos e Ricardo Ventura Santos pela revisão final do texto.

\section{RESUMO}

\section{DUARTE. M. F. S. Maturação Física: Uma}

Revisão da Literatura, com Especial Atenção à Criança Brasileira. Cad. Saúde Públ., Rio de Janeiro, 9 (suplemento 1): 7184, 1993.

O presente artigo apresenta uma revisão sobre o processo de maturação na criança em geral e discute dados em amostras brasileiras. Dados referentes às características sexuais secundárias, para ambos os sexos, são comparados aos de outras populações. A mediana de idade de menarca em meninas brasileiras, obtida pela Pesquisa Nacional de Saúde e Nutrição (Inan, 1990), está em torno de 13,2 anos. Os dados de idade de menarca em atletas brasileiras apontam para uma menarca mais tardia do que na população não-atlética. A idade de início de desenvolvimento de mamas, genitais e pêlos pubianos em crianças brasileiras difere pouco em relação a outras populações latinoamericanas e européias. Quanto à maturação sexual e às mudanças na composição corporal, vários estudos brasileiros, em concordância com dados internacionais, mostram que a menarca é um marco importante no aumento dos depósitos de gordura nas meninas. Já nos meninos, parece não haver um aumento expressivo nas dobras cutâneas durante o processo de maturação. É evidente a falta de estudos sobre crescimento, desenvolvimento e maturação do adolescente em várias regiões do país, principalmente no grupo masculino.

Palavras-Chave: Antropometria; Características Sexuais; Maturidade Sexual

\section{REFERÊNCIAS BIBLIOGRÁFICAS}

ANTUNES, J. H.; SIQUEIRA, O. B.; MENDES, O. C.; GAZZETTA, M. L. \& STANZIOLA, L., 1984. Maturação biológica em escolares de Minas Gerais - Estudo piloto. Revista B rasileira de Ciências do Esporte, 5: 21. 
AVENDANO, A. \& VALENZUELA, C., 1988. Seguimiento longitudinal de crecimiento, desarrollo de 6 a 20 anos de edad, area norte de Santiago. Pediatria, 31: 4-57.

BELMAKER, E., 1982. Sexual maturation of Jerusalem schoolgirls and its association with socioeconomic factors and ethnic groups. Annals of Human Biology, 9: 321-328.

BENITO, S. C. S.; MENDES, O. C. \& MATSUDO, V. K. R., 1983. Idade de menarca em diferentes níveis de competição no basquetebol. Revista Brasileira de Ciências do Esporte, 4: 91-94.

BEZERRA, V. L. V. A.; CAMPOS, D. \& SALOMON, J. B. R., 1973. Crescimento e desenvolvimento adolescente. Archivos Latinoamericanos de Nutrición, 23: 465-483.

BILLEWICZ, W. Z.; FELLOWES, H. M. \& THOMSON, A. M., 1981a. Menarche in Newcastle upon Tyne girls. Annals of Human Biology, 8: 313-320.

, 1981b. Pubertal changes in boys and girls in Newcastle upon Tyne. Annals of Human Biology, 8: 211-219.

BONJARDIM, E., 1984. Variações do Comprimento Tronco-Cefálico e do Comprimento de Membros Inferiores, em Função da Idade e Puberdade, em Escolares Masculinos de 10 a 19 A nos. Tese de Mestrado, São Paulo: Escola de Educação Física da Universidade de São Paulo.

BUTLER, G. E.; McKIE, M. \& RATCLIFFE, S. G., 1990. The cyclical nature of prepubertal growth. A nnals of Human Biology, 17: 177-198.

CAMERON, N.; KGAMPHE, J. S. \& LEVIN, Z., 1991. Age at menarche and analysis of secular trends in menarcheal age of South African urban and rural black females. A merican Journal of Human Biology, 3: 251-255.

CAMPOS, M. A. Z.; FRANÇA, N. M. \& MATSUDO, V. K. R., 1990. Idade de menarca em escolares de Ilha Bela. In: A nais do XVII Simpósio Internacional de Ciências do Esporte (CELAFISCS), p. 85, São Paulo: CELAFISCS.

COLLI, A. S., 1985. Maduración sexual de los adolescentes de São Paulo. In: La Salud del A dolescente y el Joven en las A méricas (OPS), pp. 249-258, Washington, DC: Organización Panamericana de la Salud. (Publicación Cientifica, 489).

CONTRERAS, M. L.; ESCOBAR, G. T.; COUPAL, N. E.; JIMENEZ, M. L. \& CASTELLANO, H. M., 1981. Estudios comparativos de la estatura y edad de la menarquia segun estrato socioeconómico en Venezuela. Archivos Latinoamericanos de Nutrición, 31: 740-757.
DACOU-VOUTETAKIS， C.; KLONTZA， D.; LAGOS, P.; TZONOU, A.; KATSAROU, E.; ANTONIADIS, S.; PAPAZISIS, G.; PAPADOULOS, G. \& MATSANIOTIS, N., 1983. Age of pubertal stages including menarche in Greek girls. A nnals of Human Biology, 10: 557-564.

DE BEM, M. F. L. \& PETROSKI, E. L., 1988. Maturação sexual em escolares de diferentes regiões climáticas. Revista Brasileira de Ciências e M ovimento, 2: 27-31.

DUARTE, C. R., 1987. Aptidão física geral de escolares de Diadema (SP) e sua relação com a maturação sexual. In: A nais do VIII Congresso Brasileiro de Medicina Desportiva, p. 16, Rio de Janeiro.

DUARTE, C. R. \& DUARTE, M. F. S., 1988. Maturação sexual e aptidão física em escolares. In: A nais do XVI Simpósio de Ciências do Esporte (CELAFISCS), p. 37, São Caetano do Sul: CELAFISCS.

, 1989. Capacidade aeróbica em escolares de 10 a 18 anos: $\mathrm{VO}_{2}$ e PWC-170. Revista Brasileira de Ciências e M ovimento, 3: 17-25.

DUARTE, M. F. S., 1986. Comportamento das Dobras Cutâneas no Processo de Maturação Sexual. Tese de Mestrado, São Paulo: Escola de Educação Física da Universidade de São Paulo. 1987. A influência da menarca na aptidão física de escolares. In: A nais do VIII Congresso Brasileiro de Medicina Desportiva, $\mathrm{p}$. 16, Rio de Janeiro.

DUARTE, M. F. S. \& DUARTE, C. R., 1992. Sexual maturation and physical fitness in Brazilian girls. In: Olympic Scientific Congress Proceedings (ICSSPE/UNISPORT), p. kin-27, MálagaSpain: UNISPORT.

DUKE, P. M.; LITT, I. F. \& GROSS, R. T., 1980. Adolescent's self-assessment of sexual maturation. Pediatrics, 66: 918-920.

EVELETH, P. B. \& TANNER, J. M., 1990. W orldwide Variation in Human Growth. Cambridge: Cambridge University Press.

FRISCH, R. E. \& REVELLE, R., 1969. Variation in body weight and the age of the adolescent growth spurt among Latin American and Asian populations in relation to calorie supplies. Human Biology, 41: 185-212. , 1970. Height and weight at menarche and a hipothesis of critical body weights and adolescent events. Science, 169: 397-399.

, 1971. Height and weight at menarche and a hipothesis of menarche. Archives of Disease in Childhood, 46: 695-701. 
FRISCH, R. E.; REVELLE, R. \& COOK, S., 1973. Components of weight at menarche and the initiation of the adolescent growth spurt in girls: Estimated total water, lean body weight and fat. Human Biology, 45: 469-483.

GALLER, J. R.; RAMSEY, F. \& SOLIMANO, G., 1985. A follow-up study of the effects of early malnutrition on subsequent development. I. Physical growth and sexual maturation during adolescence. Pediatric Research, 19: 518-523.

GASSER, T.; KNEIP, A.; ZIEGLER, P.; LARGO, R. H.; MOLINARI, L. \& PRADER, A., 1991. The dynamics of growth of width in distance, velocity, and acceleration. Annals of Human Biology, 18: 449-461.

GASSER, T.; MULLER, H. G.; KOHLER, W.; PRADER, A.; LARGO, R. H. \& MOLINARI, L., 1985. An analysis of the mid-growth and adolescent spurts of height based on acceleration. A nnals of Human Biology, 12: 129-148.

GREKSA, L. P., 1990. Age of menarche in Bolivian girls of European and Aymara ancestry. A nnals of Human Biology, 17: 49-53.

GUEDES, D. P., 1981a. Comparação somatotipológica entre escolares de diferentes níveis de maturação sexual. Revista Brasileira de Ciências do Esporte, 1 (supl.): 49.

, 1981b. Comparação dos valores de gordura subcutânea entre escolares de diferentes níveis de maturação sexual. Revista de Educação Física, 2: 21-24.

HEGG, R. V.; AMADIO, A. C.; STARK, R. E.; MANSOLDO, A. C.; KIDO, K.; TEIXEIRA, L. G. P.; BARROS, S. A. \& BASTOS, F. C., 1982. Estudo Antropométrico - Campeonato Juvenil de Atletismo - São Paulo — 1978. Revista Brasileira de Ciências do Esporte, 3: 63-79.

HEGG, R. V. \& HEGG, R., 1980. Biometria e puberdade feminina. Ars Curandi, pp. 32-44.

HEGG, R. V. \& LEVY, M. S. F., 1977. Estudo sobre a menarca - 1974. Revista da A ssociação M édica Brasileira, 23: 431-435.

HEGG, R. V. \& LUONGO, J., 1976. M edidas A ntropométricas e Desenvolvimento Pubertário em Escolares Paulistanos de 8 a 16 Anos de Idade. São Paulo: USP.

HOSHI, H. \& KOUCHI, M., 1981. Secular trend of the age at menarche of Japanese girls with special regard to the secular acceleration of the age at peak height velocity. Human Biology, 53: 593-598.

INAN (Instituto Nacional de Alimentação e Nutrição), 1990. Perfil de Crescimento da População Brasileira de 0 a 25 anos. Pesquisa Nacional sobre Saúde e Nutrição. Brasília: MS.
JOHNSTON, F. E.; ROCHE, A. F.; SCHELL, L. M. \& WETTENHALL, N. B., 1975. Critical weight at menarche critique of a hypothesis. A merican Journal of Disease in Childhood, 129: 19-23.

JORDAN, J. R., 1985. Crecimiento y Desarrollo del Adolescente: Estudio Nacional de Cuba. In: La Salud del A dolescente y el Joven en la Americas (OPAS), pp. 235-248, Washington, DC: OPAS. (Publicación Cientifica, 489)

KOZINETZ, C. A., 1988. Self-assessed female maturation stages: A research tool. Human Biology, 60: 293-303.

LARGO, R. H. \& PRADER, A., 1983a. Pubertal development in Swiss boys. Helvetica Paediatrica A cta, 38: 211-228.

LARGO, R. H. \& PRADER, A., 1983b. Pubertal development in Swiss girls. Helvetica Paediatrica A cta, 38: 229-243.

LEE, P. A., 1980. Normal ages of pubertal events among American males and females. Journal of A dolescent Health Care, 1: 26-29.

LEJARRAGA, H.; SANCHIRICO, F. U. \& CUSMINSKY, M., 1980. Age at menarche in urban Argentinian girls. A nnals of Human Biology, 7: 579-581.

LIN, W. S.; CHEN, A. C. N.; SU, J. Z. X.; ZHU, F. C.; XING, W. H.; LI, J. Y. \& YE, G. S., 1992. The menarcheal age of Chinese girls. A nnals of Human Biology, 19: 503-512.

LOPEZ, V. W.; GUILHEMOT, M.; SPYCKERELLE, V.; MULOT, B. \& DESCHAMPS, J. P., 1988. Auto-estimation des estades de maturation pubertaire chez lez adolescentes de sexe masculin. Pédiatrie, 43: 245-249.

MACMAHON, B., 1973. Age at menarche - United States. DHEW Publication No. (HRA) 74-1615, Nov. National Health Survey, Series 11, No 133.

MARSHALL, W. M. \& TANNER, J. M., 1969. Variations in the pattern of pubertal changes in girls. A rchives of Disease in Childhood, 44: 291-303.

, 1970. Variations in the pattern of pubertal changes in boys. Archives of Disease in Childhood, 45: 13-23. 1974. Puberty. In: Scientific Foundations of Pediatrics (J. A. Davis \& J. Dobbing, eds.), pp. 124-151, Philadelphia: Saunders.

MATSUDO, S. M. M. \& MATSUDO, V. K. R., 1991. Validade da auto-avaliação na determinação da maturação sexual. Revista Brasileira de Ciência e M ovimento, 5: 18-35.

MATSUDO, V. K. R., 1979. Impacto da menarca sobre valores de dobras cutâneas. In: A nais do I Congresso Brasileiro de Ciências do Esporte (CBCE), p. 50, São Caetano do Sul: CBCE. 
, 1982a. Idade de menarca em escolares da Grande São Paulo — Estudo piloto. In: A nais do X Simpósio de Ciências do Esporte (CELAFISCS), p. 19, São Caetano do Sul: CELAFISCS.

, 1982b. Menarca em esportistas brasileiras - Estudo preliminar. Revista Brasileira de Ciências do Esporte, 4: 2-6.

MATSUDO, V. K. R. \& SESSA, M., 1980. Menarca em esportistas brasileiras. In: Anais do VIII Simpósio de Ciências do Esporte (CELAFISCS), p. 34, São Caetano do Sul: CELAFISCS.

MENDES, O. C. \& MATSUDO, V. K. R., 1980. Idade de menarca em diferentes níveis de competição em atletismo. In: Anais do Congresso Regional Brasileiro de Ciências do Esporte, p. 25, Volta Redonda, Rio de Janeiro.

MOLINARI, L.; LARGO, R. H. \& PRADER, A., 1980. Analysis of the growth spurt at age seven (mid-growth spurt). Helvetica Paediatrica Acta, 35: 325-334.

MORRIS, N. M. \& UDRY, J. R., 1980. Validation of a self-administered instrument to assess stage of adolescent development. Journal of $Y$ outh A dolescence, 9: 271-280.

MUZZO, S. B.; BURROWS, R. A.; LEIVA, L. B. \& ZVAIGHAFT, A., 1988. Características de la puberdad de escolares de sexo masculino de diferentes niveles socioeconómicos de la región metropolitana de Chile. Revista Chilena de Pediatria, 59: 240-246.

NIELSEN, C. T.; SKAKKAEB, N. E.; RICHARDSON, D. W.; DARLING, J. A. B.; HUNTER, W. M.; JORGENSEN, M.; NIELSEN, A.; INGERSLEV, O.; KEIDING, N. \& MULLER, J., 1986. Onset of the release of spermatozoa (spermarche) in boys in relation to age, testicular growth, pubic hair, and height. Journal of Clinical Endocrinology and M etabolism, 62: 532-535.

PAWSON, I. G., 1977. Growth characteristics of populations of Tibetan origin in Nepal. A merican Journal of Physical Anthropology, 47: 473-482.

PETROSKI, E. L.; DUARTE, M. F. S. \& MATSUDO, V. K. R., 1983. Idade de menarca em escolares catarinenses. Revista de Educação Física, 4: 3-6.

PRADER, A., 1966. Testicular size: Assessment and clinical importance. Triangle, 7: 240-243.

REYNOLDS, E. L. \& WINES, J. V., 1948. Individual differences in physical changes associated with adolescence in girls. A merican Journal of Disease of Children, 75: 329-350.

, 1951. Physical changes associated with adolescence in boys. American Journal of Disease of Children, 82: 529-547.
RIEHMER, C. \& VIOLATO, P. R. S., 1983. Idade de menarca em escolares de Londrina-PR. Revista B rasileira de Ciências do Esporte, 5: 21.

ROCHE, A. F.; TYLESHEVSKI, F. \& ROGERS, E., 1983. Non-invasive measurements of physical maturity in children. Research Quarterly for Exercise and Sport, 54: 364-371.

SAITO, M. I., 1984. Maturação sexual: Auto-avaliação do adolescente. Pediatria, 6: 111-115.

1985. Menarca e Variáveis A ntropométricas em Adolescentes Brasileiras. Tese de Mestrado, São Paulo: Faculdade de Medicina da Universidade de São Paulo.

SATYANARAYANA, K. \& NADAMUNI NAIDU, A., 1979. Nutrition and menarche in rural Hyderabad. A nnals of Human Biology, 6: 163-165.

SATYANARAYANA, K.; NADAMUNI NAIDU, A. \& NARASINGA RAO, B. S., 1980. Adolescent growth spurt among rural Indian boys in relation to their nutritional status in early childhood. A nnals of Human Biology, 7: 359-365.

SEMPE, M.; PECHON, G. \& PERNOT, M. P. R., 1979. A uxologie: Méthode et Séquences. Paris: Ed. Laboratorie Theraplix.

SILVA, S. R.; MENDES, O. C. \& MATSUDO, V. K. R., 1984. Avaliação da maturação biológica de nadadoras. In: Anais do XII Simpósio de Ciências do Esporte (CELAFISCS), p. 40, São Caetano do Sul, CELAFISCS.

SILVA, T. M. A.; NASCIMENTO, D. V.; SILVA, P. T. N. \& MATSUDO, V. K. R., 1982. Idade de menarca das escolares maranhenses. In: A nais do X Simpósio de Ciências do Esporte (CELAFISCS), p. 17, São Caetano do Sul: CELAFISCS.

TANNER, J. M., 1962. Growth at Adolescence. Oxford: Blackwell Scientific Publications.

TARANGER, J.; ENGSTROM, I.; LICHTENSTEIN, H. \& REDEGREN, I. S., 1976. VI Somatic pubertal development. Acta Paediatrica Scandinavica, 258 (suppl.): 121-135.

TSE, W. Y.; HINDMARSH, P. C. \& BROOK, C. G. D., 1989. The infancy-childhood puberty model of growth: Clinical aspects. Acta Paediatrica Scandinavica, 356 (suppl.): 38-45.

VAN WIERINGEN, J. C.; WAFELBAKKER, F.; VERBRUGGE, H. P. \& DE HASS, J. M., 1971. Growth Diagrams, 1965, Netherlands. Leiden: Netherlands Institute of Preventive Medicine.

VILLARREAL, S. F.; MARTORELL, R. \& MENDOZA, F., 1989. Sexual maturation of MexicanAmerican adolescents. Annals of Human Biology, 1: 87-95. 
VIOLATO, P. R. S. \& MATSUDO, V. K. R., 1983. Menarca em escolares da rede de ensino de Rolândia-PR. Revista Brasileira de Ciências do Esporte, 5: 29.

VÍVOLO, M. A.; SILVA, S. A. P. S.; PERAZZOLO, S. \& MATSUDO, V. K. R., 1983. Avaliação da maturação sexual e características antropométricas de atletas da Seleção Paulista de Ginástica Rítmica Desportiva. Revista Brasileira de Ciências do Esporte, 5: 33.
ZACHMAN, M.; PRADER, A.; KIND, H. P.; HAFLIGER, H. \& BUDLIGER, H., 1974. Testicular volume during adolescence. Cross-sectional and longitudinal studies. Helvetica Paediatrica A cta, 29: 61-72. 